Research Article

\title{
Exploration of Unsteady Squeezing Flow through Least Squares Homotopy Perturbation Method
}

\author{
Mubashir Qayyum (i) and Imbsat Oscar \\ National University of Computer and Emerging Sciences-FAST Lahore Campus, Lahore, Pakistan \\ Correspondence should be addressed to Mubashir Qayyum; mubashir.qayyum@nu.edu.pk
}

Received 28 May 2021; Revised 8 July 2021; Accepted 24 August 2021; Published 6 September 2021

Academic Editor: Basil Papadopoulos

Copyright (c) 2021 Mubashir Qayyum and Imbsat Oscar. This is an open access article distributed under the Creative Commons Attribution License, which permits unrestricted use, distribution, and reproduction in any medium, provided the original work is properly cited.

\begin{abstract}
Squeezing flow has many applications in different fields including chemical, mechanical, and electrical engineering as these flows can be observed in many hydrodynamical tools and machines. Due to importance of squeezing flow, in this paper, an unsteady squeezing flow of a viscous magnetohydrodynamic (MHD) fluid which is passing through porous medium has been modeled and analyzed with and without slip effects at the boundaries. The least squares homotopy perturbation method (LSHPM) has been proposed to determine the solutions of nonlinear boundary value problems. To check the validity and convergence of the proposed scheme (LSHPM), the modeled problems are also solved with the Fehlberg-Runge-Kutta method (RKF45) and homotopy perturbation method (HPM) and residual errors are compared with LSHPM. To the best of the authors' knowledge, the current problems have not been attempted before with LSHPM. Moreover, the impact of different fluid parameters on the velocity profile has been examined graphically in slip and no-slip cases. Analysis shows that the Reynolds number, MHD parameter, and porosity parameter have opposite effects in case of slip and no slip at the boundaries. It is also observed that nonzero slip parameter accelerates the velocity profile near the boundaries. Analysis also reveals that LSHPM provides better results in terms of accuracy as compared to HPM and RKF45 and can be effectively used for the fluid flow problems.
\end{abstract}

\section{Introduction}

In squeezing flow, fluid is squeezed between two parallel objects. This phenomenon can be observed in many hydrodynamical machines and tools. One can observe the application of these flows in food industry and chemical engineering [1-4]. Few common examples are polymer handling, compression and injection molding, and modeling of lubrications. Modeling of squeezing flow was initiated around nineteenth century, and from then onward, it got attention because of its endless implementations in various fields. The initial studies in these flows were done by Stefan [5], and he worked on Newtonian fluid and found an asymptotic solution.

The study of fluids with electromagnetic field is an exceptionally interesting area of magnetohydrodynamics (MHD). Utilizing MHD fluid as lubricant is intriguing, since under certain extreme conditions, it averts the unpredicted change of lubricant viscosity with temperature. Maki et al. [6] investigated the MHD fluid as lubricant in an externally pressurized thrust bearing. Different authors have examined the effects of magnetic field in the flow of fluids [7-10]. The squeezing flow between two disks together with the presence of a magnetic field was studied in [11], and that between the rotating disks was studied in $[12,13]$. A material that has fluid-filled pores is known as porous medium. It is described by properties which are permeability and porosity. The amount of fluid which passes through the porous medium is known as permeability, while porosity is characterized as the sum of fluid held by the fabric. Porous medium has several applications in the field of petroleum, reservoir, and chemical engineering [14-18].

Slip is difficult to calculate or observe directly in a porous medium [19]. Thus, most observations rely upon theoretical analysis and numerical methods. The affirmation of slip has been confirmed experimentally through indirect approaches 
[20]. Considering slip results in increasing error and computational time, which is why it is ignored in many theoretical analyses [21, 22].

At a fluid-solid interface, the effect of slip is an established boundary condition. It has various applications in science and technology, for instance, material processing, rheometric measurements, and fluid transportation [20,23]. The boundary condition of a viscous fluid at a macroscopic level is considered to be no-slip condition [21] which means that the instantaneous velocity would be zero at the boundary. Nonetheless, noninstantaneous slip conditions because of shear stress have also been investigated by the implication of unexpected decrease in viscosity or increase in flow rates [20]. This slip condition is known as the apparent slip in fluid mechanics.

Mooney presented the pioneering work in slip analysis [24]. He determined explicit formulas for fluidity and slip. The techniques for dealing with slip condition are significant for investigation of fluid flow; however, it is generally underestimated or ignored in the investigations including complex fluid systems [25].

In this paper, numerical and seminumerical solutions of an unsteady squeezing flow of viscous fluid with MHD and porosity effects have been analyzed with slip and no-slip conditions at the boundary. Problems have been solved through LSHPM which is the modification of well-known HPM [26]. LSHPM is the coupling of HPM along with least squares optimizer [27-29]. The main feature of LSHPM is accelerated convergence with less computational cost. For validation of the LSHPM results, these problems were also solved with HPM and Fehlberg-Runge-Kutta method (RKF45), and the results were compared with LSHPM. Error analysis is performed in this study via tables and graphs. The remaining sections are organized as follows. Mathematical formulation is given in Section 2. The basic concept of LSHPM is given in Section 3. The application of LSHPM on modeled problems is given in Section 4. Results and discussion are given in Section 5. Finally, the conclusion is presented in Section 6.

\section{Problem Formulation}

Let us consider a rectilinear, unsteady magnetohydrodynamic squeezing flow of an incompressible viscous fluid passing through a porous medium between two infinite parallel plates. The distance between the plates is always $2 \alpha(t)$ at any time $t$. The $x$-axis is considered the central axis of the channel, and the $y$-axis is perpendicular to the channel. $\beta=\beta\left(0, \beta_{0}, 0\right)$ is a uniform magnetic field that acts along the $y$-axis. The induced magnetic field is considered negligible, and the direction of the magnetic field is perpendicular to the flow of the fluid and has a constant strength $H_{0}$. In fact, $\beta_{0}=H_{0} \mu_{0}$, where $\mu_{0}$ is the magnetic permeability. Furthermore, the plate is considered to move symmetrically about the central axis of the channel.

Following are the mass and momentum conservation equations presenting the unsteady flow [30]:

$$
\begin{gathered}
\frac{\partial \widetilde{u}}{\partial x}+\frac{\partial \widetilde{v}}{\partial y}=0 \\
\rho\left(\frac{\partial \widetilde{u}}{\partial t}+\widetilde{u} \frac{\partial \widetilde{u}}{\partial x}+\widetilde{v} \frac{\partial \widetilde{u}}{\partial y}\right)=-\frac{\partial p}{\partial x}+v\left(\frac{\partial^{2} \widetilde{u}}{\partial x^{2}}+\frac{\partial^{2} \widetilde{u}}{\partial y^{2}}\right)-\sigma \beta_{0}^{2} \widetilde{u}-\frac{\mu}{k} \widetilde{u} \\
\rho\left(\frac{\partial \widetilde{v}}{\partial t}+\widetilde{u} \frac{\partial \widetilde{v}}{\partial x}+\widetilde{v} \frac{\partial \widetilde{v}}{\partial y}\right)=-\frac{\partial p}{\partial y}+v\left(\frac{\partial^{2} \widetilde{v}}{\partial x^{2}}+\frac{\partial^{2} \widetilde{v}}{\partial y^{2}}\right)-\frac{\mu}{k} \widetilde{v}
\end{gathered}
$$

where $\widetilde{u}$ and $\widetilde{v}$ are the velocity components along $x$-axis and $y$-axis, respectively, and $\sigma, \rho$, and $\nu$ represent the electric conductivity, density, and kinematic viscosity, respectively. Let us define the vorticity function $\widetilde{\omega}$ as

$$
\widetilde{\omega}=\frac{\partial \widetilde{v}}{\partial x}-\frac{\partial \widetilde{u}}{\partial y},
$$

and the generalized pressure $h$ as

$$
h=\frac{\rho}{2}\left(\widetilde{u}^{2}+\widetilde{v}^{2}\right)+p .
$$

By plugging (4) and (5) in (1)-(3), the above mass and momentum equations turn out be

$$
\begin{gathered}
\frac{\partial \widetilde{u}}{\partial x}+\frac{\partial \widetilde{v}}{\partial y}=0 \\
\frac{\partial h}{\partial x}+\rho\left(\frac{\partial \widetilde{u}}{\partial t}-\widetilde{v} \widetilde{\omega}\right)=-\nu \frac{\partial \widetilde{\omega}}{\partial y}-\sigma \beta^{2} \widetilde{u}-\frac{\mu}{k} \widetilde{u} \\
\frac{\partial h}{\partial y}+\rho\left(\frac{\partial \widetilde{v}}{\partial t}+\widetilde{u} \widetilde{\omega}\right)=\nu \frac{\partial \widetilde{\omega}}{\partial x}-\frac{\mu}{k} \widetilde{v} .
\end{gathered}
$$

After eliminating pressure gradient using (7) and (8), the following is obtained:

$$
\rho\left(\frac{\partial \widetilde{\omega}}{\partial t}+\left(\widetilde{u} \frac{\partial}{\partial x}+\widetilde{v} \frac{\partial}{\partial y} \widetilde{\omega}\right)\right)=\nu \nabla^{2} \widetilde{\omega}-\sigma \beta^{2} \frac{\partial \widetilde{u}}{\partial y}-\frac{\mu}{k} \widetilde{\omega},
$$

along with boundary conditions

$$
\begin{aligned}
y & =\alpha, \\
\widetilde{u}(x, y, t) & =0, \\
\widetilde{v}(x, y, t) & =\widetilde{u}_{w}(t), \\
y & =0, \\
\widetilde{v}(x, y, t) & =0, \\
\frac{\partial \widetilde{u}(x, y, t)}{\partial y} & =0,
\end{aligned}
$$

where $\widetilde{v}_{w}(t)=\mathrm{d} \alpha / \mathrm{d} t$ represents the velocity of plates. Let a dimensionless variable $\xi=y / \alpha(t)$, where $2 \alpha(t)$ is the distance between the plates at any time $t$. Here equations (6) and (9) have the following form: 


$$
\begin{gathered}
\frac{\partial \widetilde{u}}{\partial x}+\frac{\partial \widetilde{v}}{\alpha(t) \partial y}=0 \\
\rho\left(\frac{\partial \widetilde{\omega}}{\partial t}+\left(\widetilde{u} \frac{\partial}{\partial x}+\widetilde{v} \frac{\partial}{\alpha(t) \partial \xi} \widetilde{\omega}\right)\right)=\nu \nabla^{2} \widetilde{\omega}-\sigma \beta_{0}^{2} \frac{\partial}{\alpha(t) \partial \xi}-\frac{\mu}{k} \widetilde{\omega} .
\end{gathered}
$$

On $\widetilde{u}(x, \xi, t)$ and $\widetilde{v}(x, \xi, t)$, the boundary conditions are as follows:

$$
\begin{aligned}
\text { at } \quad \xi & =1, \\
\widetilde{u}(x, \xi, t) & =0, \\
\widetilde{v}(x, \xi, t) & =v_{w}(t), \\
\text { at } \quad \xi & =0, \\
\widetilde{u}(x, \xi, t) & =0, \\
\frac{\partial \widetilde{u}(x, \xi, t)}{\partial \xi} & =0 .
\end{aligned}
$$

Velocity components are defined as $[30,31]$

$$
\begin{aligned}
\widetilde{u} & =\frac{K-x_{0}}{\alpha(t)} \widetilde{v}_{w} f^{\prime}(\xi), \\
\widetilde{v} & =\widetilde{v}_{w}(t) f(\xi) .
\end{aligned}
$$

By plugging (16) in (12) and (13), the continuity equation is identically satisfied and (13) becomes

$$
\begin{aligned}
& \frac{\alpha \widetilde{v}_{w}}{v}\left(f f^{\prime \prime \prime}-f^{\prime} f^{\prime \prime}-\xi f D^{\prime \prime \prime}-2 f^{\prime \prime}\right)+\frac{\alpha^{2}}{\nu \widetilde{v}_{w}} \frac{\mathrm{d} \widetilde{v}_{w}}{\mathrm{~d} t} f^{\prime \prime} \\
= & f^{i v}-f^{\prime \prime} M_{g}-M_{p} f^{\prime \prime},
\end{aligned}
$$

where the primes are representing the derivative of $f$ with respect to $\xi$ and $M_{g}$ and $M_{p}$ are the MHD parameter and the porosity parameter, respectively. We determine the boundary conditions from (14)-(16):

$$
\begin{aligned}
f(1) & =1, \\
f^{\prime}(1) & =0, \\
f(0) & =0, \\
f^{\prime \prime}(0) & =0 .
\end{aligned}
$$

Therefore, for a similarity solution, let us define the following:

$$
\begin{gathered}
\frac{\alpha \widetilde{v}_{w}}{v}=R, \\
\frac{\alpha^{2}}{v \widetilde{v}_{w}} \frac{\mathrm{d} \widetilde{v}_{w}}{\mathrm{~d} t}=R Q,
\end{gathered}
$$

where $R$ and $Q$ are the functions of $t$, but these functions are taken to be constants for a similarity solution. By integrating $\alpha \widetilde{v}_{w} / \nu=R$, we obtain

$$
\alpha(t)=\left(2 \nu R t+\alpha_{0}^{2}\right)^{1 / 2} .
$$

It follows from (13) and (14) that $Q=-1$, so (10) becomes

$$
f^{i v}-R\left[f f^{\prime \prime \prime}-f^{\prime} f^{\prime \prime}-\frac{\xi}{f^{\prime \prime \prime}}-3 f^{\prime \prime}\right]-M_{g} f^{\prime \prime}-M_{p} f^{\prime \prime}=0,
$$

where $R$ denotes the Reynolds number. The boundary conditions in case of no slip subject to (18) and (19) are

$$
\begin{aligned}
f(1) & =1, \\
f^{\prime}(1) & =0, \\
f(0) & =0, \\
f^{\prime \prime}(0) & =0,
\end{aligned}
$$

and in case of slip at the boundary, the corresponding conditions are

$$
\begin{aligned}
f(1) & =1, \\
f^{\prime}(1) & =\gamma f^{\prime \prime}(1), \\
f(0) & =0, \\
f^{\prime \prime}(0) & =0,
\end{aligned}
$$

where $\gamma$ is the slip parameter.

\section{Basic Idea of Least Squares Homotopy Perturbation Method}

Let us consider a general differential equation

$$
\begin{aligned}
L(\varphi)+N(\varphi)-g(r) & =0, \quad r \in \Omega, \\
B\left(\varphi, \frac{\mathrm{d}^{n} \varphi}{\mathrm{d} r^{n}}\right) & =0, \quad r \in \gamma,
\end{aligned}
$$

where $L, N$, and $B$ represent the linear and nonlinear parts and boundary operator, respectively, and $\varphi$ represents an unknown function while $g(x)$ is known function.

First of all, we construct a homotopy for (25) such that $\eta(r, p): \varphi \times[0,1] \longrightarrow \mathbb{R}$ which satisfies

$$
\begin{aligned}
\psi(\eta, p) & =(1-p)\left[L(\eta)-L\left(\varphi_{0}\right)\right]+p[L(\eta)+N(\eta)-g(r)] \\
& =0, \quad r \in \Omega,
\end{aligned}
$$

where $p \in[0,1]$ is the embedding parameter and $L\left(\varphi_{0}\right)$ is the initial guess.

As $p$ varies from 0 to 1 , then $\varphi_{0}$ approaches $\tilde{\varphi}(r)$. Taylor series expansion of $\eta(r, p)$ about $p$ is 


$$
\eta(r, p)=\eta_{0}+\sum_{k=1}^{\infty} \eta_{k} p^{k}
$$

Setting $p=1$, the approximate solution of (25) would be

$$
\widetilde{\varphi}=\lim _{p \longrightarrow 1} \eta(r, p)=\sum_{k=1}^{\infty} \eta_{k} .
$$

After reassigning the dummy coefficients $c_{i^{\prime}} s$ in obtained series solution (29), we substitute the approximate solution $\widetilde{\varphi}$ back in equation (25) to get the residual function:

$$
\mathbb{R}\left(x, c_{i}\right)=R(x, \tilde{\varphi}) .
$$

Now we will compute the sum of squares of residuals:

$$
\mathbb{J}\left(c_{i}\right)=\int_{I} \mathbb{R}^{2}\left(x, c_{i}\right) \mathrm{d} x .
$$

After computing $J\left(c_{i}\right)$, we find the optimal values of $c_{i}^{\prime} s$ from system of equations obtained from $\partial J / \partial c_{i}=0$.

Putting these optimal values back into series (29), we will get our final series form solution of LSHPM.

\section{Application of LSHPM to Squeezing Flow}

Let us construct a homotopy for the given problem as follows:

$$
\begin{aligned}
& (1-p)\left(f^{i v}\right)+p\left(f^{i v}-R\left[f f^{\prime \prime}-f^{\prime} f^{\prime \prime}-\xi f^{\prime \prime}-3 f^{\prime \prime}\right]\right. \\
& \left.-M_{g} f^{\prime \prime}-M_{p} f^{\prime \prime}\right)=0 .
\end{aligned}
$$

Using (32) and (23), various order problems along with solutions are given as follows.

The zeroth-order problem is

$$
\begin{gathered}
f_{0}^{(i v)}(\xi)=0 \\
f_{0}(1)=1 \\
f_{0}^{\prime}(1)=0 \\
f_{0}(0)=0 \\
f_{0}^{\prime \prime}(0)=0 .
\end{gathered}
$$

The solution of (33) is

$$
f_{0}(\xi)=\frac{1}{2}\left(3 \xi-\xi^{3}\right)
$$

The first-order problem is

$$
\begin{aligned}
f_{1}^{(\mathrm{iv})}(\xi) & =M_{p} f_{0}^{\prime \prime}(\xi)+M_{g} f_{0}^{\prime \prime}(\xi)-3 R f_{0}^{\prime \prime}(\xi)-R f_{0^{\prime}}(\xi) f_{0}^{\prime \prime}(\xi)-R \xi f_{0}^{\prime \prime \prime}(\xi)+R f_{0}(\xi) f_{0}^{\prime \prime \prime}(\xi) \\
f_{1}(1) & =0 f_{1}^{\prime}(1)=0, f_{1}(0)=0, f_{1}^{\prime \prime}(0)=0 .
\end{aligned}
$$

The solution of (35) is

$$
f_{1}(\xi)=\frac{1}{280}\left(-7 M_{p} \xi-7 M_{g} \xi+26 R \xi+14 M_{p} \xi^{3}+14 M_{g} \xi^{3}-53 R \xi^{3}-7 M_{p} \xi^{5}-7 M_{g} \xi^{5}+28 R \xi^{5}-R \xi^{7}\right)
$$

The second-order problem is

$$
\begin{aligned}
f_{2}^{(\mathrm{iv})}(\xi)= & -R f_{1}^{\prime}(\xi) f_{0}^{\prime \prime}(\xi)+M_{p} f_{1}^{\prime \prime}(\xi)+M_{g} G_{1}^{\prime \prime}(\xi)-3 R f_{1}^{\prime \prime}(\xi)-R f_{0}^{\prime}(\xi) f_{1}^{\prime \prime}(\xi)+R f_{1}(\xi) f_{0}^{\prime \prime \prime}(\xi)+R x f_{1}^{\prime \prime \prime}(\xi) \\
& +R f_{0}(\xi) f_{1}^{\prime \prime \prime}(\xi) \\
f_{2}(1)= & 0, f_{2}^{\prime}(1)=0, f_{2}(0)=0, f_{2}^{\prime \prime}(0)=0
\end{aligned}
$$

The solution (37) is 


$$
\begin{aligned}
f_{2}(\xi)= & \frac{1}{7761600}\left(10164 M_{p}^{2} \xi+20328 M_{p} M_{g} \xi+10164 M_{g}^{2} \xi-78771 M_{p} M_{g} \xi-78771 M_{g} R \xi+152862 R^{2} \xi\right. \\
& -24948 M_{p}^{2} \xi^{3}-49896 M_{p} M_{g} \xi^{3}-24948 M_{g}^{2} \xi^{3}+193116 M_{p} M_{g} \xi^{3}+193116 M_{p} M_{g} \xi^{3}-374200 R^{2} \xi^{3} \\
& +19404 M_{p}^{2} \xi^{5}+38808 M_{p} M_{g} \xi^{5}+19404 M_{g}^{2}-151074 M_{p} R \xi^{5}-151074 M_{g} R \xi^{5}+293832 R^{2} \xi^{5} \\
& -4620 M_{p} \xi^{7}-9240 M_{p} M_{g} \xi^{7}-4620 M_{g}^{2} \xi^{7}+37884 M_{p} R \xi^{7}+37884 M_{g} R \xi^{7}-76428 R^{2} \xi^{7} \\
& \left.-1155 M_{p} R \xi^{9}-1155 M_{g} R \xi^{9}+3850 R^{2} \xi^{9}+84 R^{2} \xi^{11}\right) .
\end{aligned}
$$

Now, $\sum_{i=0}^{2} f_{i}(\xi)$ gives a series form solution $f(\xi)$ as

$$
\begin{aligned}
f(\xi)= & \frac{1}{2}\left(3 \xi-\xi^{3}\right)+\frac{1}{280}\left(-7 M_{p} \xi-7 M_{g} \xi+26 R \xi+14 M_{p} \xi^{3}+14 M_{g} \xi^{3}\right. \\
& \left.-53 R \xi^{3}-7 M_{p} \xi^{5}-7 M_{g} \xi^{5}+28 R \xi^{5}-R \xi^{7}\right)+\frac{1}{7761600}\left(10164 M_{p}^{2} \xi+20328 M_{p} M_{g} \xi+10164 M_{g}^{2} \xi\right. \\
& -78771 M_{p} M_{g} \xi-78771 M_{g} R \xi+152862 R^{2} \xi-24948 M_{p}^{2} \xi^{3}-49896 M_{p} M_{g} \xi^{3}-24948 M_{g}^{2} \xi^{3} \\
& +193116 M_{p} M_{g} \xi^{3}+193116 M_{p} M_{g} \xi^{3}-374200 R^{2} \xi^{3}+19404 M_{p}^{2} \xi^{5}+38808 M_{p} M_{g} \xi^{5}+19404 M_{g}^{2} \\
& -151074 M_{p} R \xi^{5}-151074 M_{g} R \xi^{5}+293832 R^{2} \xi^{5}-4620 M_{p} \xi^{7}-9240 M_{p} M_{g} \xi^{7}-4620 M_{g}^{2} \xi^{7} \\
& \left.+37884 M_{p} R \xi^{7}+37884 M_{g} R \xi^{7}-76428 R^{2} \xi^{7}-1155 M_{p} R \xi^{9}-1155 M_{g} R \xi^{9}+3850 R^{2} \xi^{9}+84 R^{2} \xi^{11}\right) .
\end{aligned}
$$

It follows that (39) consists of $\xi, \xi^{3}, \xi^{5}, \xi^{7}, \xi^{9}, \xi^{11}$. Let us assume that

$$
\tilde{f}(\xi)=c_{1} x+c_{2} \xi^{3}+c_{3} \xi^{5}+c_{4} \xi^{7}+c_{5} \xi^{9}+c_{6} \xi^{11}
$$

Now, we find the optimal coefficients of $\xi_{i}^{\prime} s$ by using the least squares optimizer. By applying boundary conditions (23), the problem reduces to the following form:

$$
\begin{aligned}
& c_{1}=1-\left(c_{2}+c_{3}+c_{4}+c_{5}+c_{6}\right), \\
& c_{2}=-\frac{1}{2}\left(1+4 c_{3}+6 c_{4}+8 c_{5}+10 c_{6}\right) .
\end{aligned}
$$

$$
\tilde{f}(\xi)=t\left(1-c_{3}-c_{4}-c_{5}-c_{6}+\frac{1}{2}\left(1+4 c_{3}+6 c_{4}+8 c_{5}+10 c_{6}\right)\right) \xi+\frac{1}{2}\left(-1-4 c_{3}-6 c_{4}-8 c_{5}-10 c_{6}\right) \xi^{3}+c_{3} \xi^{5}+c_{4} \xi^{7}+c_{5} \xi^{9}+c_{6} \xi^{11}
$$

Next, putting $\tilde{f}(\xi)$ in (22) will gives us the following residual function.

After computing $J\left(c_{3}, c_{4}, c_{5}, c_{6}\right)=\int_{0}^{1} R^{2}\left(c_{3}, c_{4}, c_{5}, c_{6}\right) \mathrm{d} \xi$, optimal values of $c_{i}^{\prime} s$ can be obtained from $\partial J / \partial c_{i}=0$.

The same procedure is applied to (22) and (24) to calculate the second-order solution in case of slip at the boundary.

\section{Results and Discussion}

In this article, an unsteady squeezing flow of MHD fluid passing through a porous medium with slip and no slip at the boundary has been examined through various methods including LSHPM, HPM, and RKF45.

The problems have been solved for various values of fluid parameters in case of slip and no slip at the boundary. Validity of the obtained solutions through LSHPM has been confirmed by comparing results with numerical schemes (RKF45) and a semianalytical scheme (HPM). This can also be observed from Tables 1 and 2 in case of no slip and Tables 3 and 4 in case of slip at the boundary. These tables signify the efficiency of LSHPM and clearly show that the results obtained from LSHPM are better in terms of accuracy along with less computational cost. 
TABLE 1: Solutions along with residual errors for RKF45, HPM, and LSHPM when $R=0.5, M_{g}=1$, and $M_{p}=0.7$ in case of no slip.

\begin{tabular}{lcccccc}
\hline$\xi$ & \multicolumn{2}{c}{ Fehlberg RK } & \multicolumn{2}{c}{ HPM } & \multicolumn{2}{c}{ LSHPM } \\
& Solution & Error & Solution & Error & Solution & Error \\
\hline 0. & 0. & $3.4693 \times 10^{-3}$ & 0. & 0. & 0. & 0.149891 \\
0.1 & 0.149891 & $5.0355 \times 10^{-4}$ & 0.149891 & $3.42652 \times 10^{-5}$ & $1.56717 \times 10^{-7}$ \\
0.2 & 0.296726 & $7.76524 \times 10^{-5}$ & 0.296726 & $6.52833 \times 10^{-5}$ & 0.296726 & $3.04651 \times 10^{-7}$ \\
0.3 & 0.437456 & $3.7471 \times 10^{-5}$ & 0.437456 & $8.99533 \times 10^{-5}$ & 0.437456 & $2.8858 \times 10^{-7}$ \\
0.4 & 0.56905 & $2.18501 \times 10^{-5}$ & 0.56905 & $1.05351 \times 10^{-4}$ & 0.56905 & $5.63137 \times 10^{-8}$ \\
0.5 & 0.688501 & $2.01114 \times 10^{-5}$ & 0.6885 & $1.08865 \times 10^{-4}$ & 0.688501 & $5.35202 \times 10^{-7}$ \\
0.6 & 0.792825 & $5.14344 \times 10^{-6}$ & 0.792825 & $9.90945 \times 10^{-5}$ & 0.792825 & $4.88741 \times 10^{-7}$ \\
0.7 & 0.879069 & $5.4068 \times 10^{-5}$ & 0.879068 & $7.82969 \times 10^{-5}$ & 0.879069 & $4.36404 \times 10^{-7}$ \\
0.8 & 0.944296 & $6.14172 \times 10^{-5}$ & 0.944296 & $5.62981 \times 10^{-5}$ & 0.944296 & $9.08635 \times 10^{-7}$ \\
0.9 & 0.985583 & $4.71814 \times 10^{-4}$ & 0.985583 & $5.30898 \times 10^{-5}$ & 0.985583 & $1.06586 \times 10^{-6}$ \\
1. & 1. & $3.11918 \times 10^{-3}$ & 1. & $9.17254 \times 10^{-5}$ & 1. & $3.97623 \times 10^{-6}$ \\
\hline
\end{tabular}

TABLE 2: Solutions along with residual errors for RKF45, HPM, and LSHPM when $R=1, M_{g}=1$, and $M_{p}=0.3$ in case of no slip.

\begin{tabular}{|c|c|c|c|c|c|c|}
\hline \multirow{2}{*}{$\xi$} & \multicolumn{2}{|c|}{ Fehlberg RK } & \multicolumn{2}{|c|}{ HPM } & \multicolumn{2}{|c|}{ LSHPM } \\
\hline & Solution & Error & Solution & Error & Solution & Error \\
\hline 0. & 0. & $2.58588 \times 10^{-2}$ & 0 & 0 & 0. & 0 \\
\hline 0.1 & 0.156416 & $1.94025 \times 10^{-3}$ & 0.156262 & $3.36615 \times 10^{-2}$ & 0.156416 & $5.4093 \times 10^{-5}$ \\
\hline 0.2 & 0.30896 & $5.70757 \times 10^{-4}$ & 0.308676 & $6.23969 \times 10^{-2}$ & 0.30896 & $6.35526 \times 10^{-5}$ \\
\hline 0.3 & 0.453866 & $2.11488 \times 10^{-4}$ & 0.453494 & $8.19041 \times 10^{-2}$ & 0.453866 & $1.74718 \times 10^{-5}$ \\
\hline 0.4 & 0.587568 & $5.92517 \times 10^{-5}$ & 0.587161 & $8.90784 \times 10^{-2}$ & 0.587568 & $4.80029 \times 10^{-5}$ \\
\hline 0.5 & 0.706796 & $2.15368 \times 10^{-4}$ & 0.706409 & $8.24848 \times 10^{-2}$ & 0.706796 & $7.03709 \times 10^{-5}$ \\
\hline 0.6 & 0.808646 & $1.07431 \times 10^{-4}$ & 0.808327 & $6.26809 \times 10^{-2}$ & 0.808646 & $1.40914 \times 10^{-5}$ \\
\hline 0.7 & 0.890646 & $1.38249 \times 10^{-4}$ & 0.890426 & $3.23326 \times 10^{-2}$ & 0.890646 & $7.08122 \times 10^{-5}$ \\
\hline 0.8 & 0.950797 & $7.04565 \times 10^{-4}$ & 0.950682 & $3.95662 \times 10^{-3}$ & 0.950797 & $5.19128 \times 10^{-5}$ \\
\hline 0.9 & 0.987588 & $2.49995 \times 10^{-3}$ & 0.987555 & $4.02371 \times 10^{-2}$ & 0.987588 & $9.77666 \times 10^{-5}$ \\
\hline 1. & 1. & $2.86845 \times 10^{-2}$ & 1. & $7.04387 \times 10^{-2}$ & 1. & $2.68303 \times 10^{-4}$ \\
\hline
\end{tabular}

TABLE 3: Solutions along with residual errors for RKF45, HPM, and LSHPM when $R=0.5, M_{g}=1, M_{p}=0.8$, and $\gamma=0.9$ in case of slip.

\begin{tabular}{|c|c|c|c|c|c|c|}
\hline \multirow{2}{*}{$\xi$} & \multicolumn{2}{|c|}{ Fehlberg RK } & \multicolumn{2}{|c|}{ HPM } & \multicolumn{2}{|c|}{ LSHPM } \\
\hline & Solution & Error & Solution & Error & Solution & Error \\
\hline 0. & 0 & $1.51816 \times 10^{-3}$ & 0 & 0 & 0. & 0 \\
\hline 0.1 & 0.0693885 & $1.12203 \times 10^{-4}$ & 0.0693931 & $1.17989 \times 10^{-4}$ & 0.0693885 & $5.75455 \times 10^{-7}$ \\
\hline 0.2 & 0.140654 & $2.57789 \times 10^{-5}$ & 0.140663 & $2.51814 \times 10^{-4}$ & 0.140654 & $6.52198 \times 10^{-7}$ \\
\hline 0.3 & 0.21567 & $8.08689 \times 10^{-6}$ & 0.215683 & $4.13354 \times 10^{-4}$ & 0.21567 & $1.39485 \times 10^{-7}$ \\
\hline 0.4 & 0.296303 & $2.76334 \times 0^{-6}$ & 0.296317 & $6.07337 \times 10^{-4}$ & 0.296303 & $5.24535 \times 10^{-7}$ \\
\hline 0.5 & 0.384403 & $4.29423 \times 10^{-6}$ & 0.384419 & $8.29514 \times 10^{-4}$ & 0.384403 & $6.79866 \times 10^{-7}$ \\
\hline 0.6 & 0.481805 & $8.05261 \times 10^{-6}$ & 0.481821 & $1.06662 \times 10^{-3}$ & 0.481805 & $6.10725 \times 10^{-8}$ \\
\hline 0.7 & 0.590316 & $4.58094 \times 10^{-5}$ & 0.590331 & $1.29826 \times 10^{-3}$ & 0.590316 & $7.03765 \times 10^{-7}$ \\
\hline 0.8 & 0.71171 & $8.15773 \times 10^{-5}$ & 0.711721 & $1.50056 \times 10^{-3}$ & 0.71171 & $3.9953 \times 10^{-7}$ \\
\hline 0.9 & 0.847715 & $6.82106 \times 10^{-4}$ & 0.847721 & $1.65152 \times 10^{-3}$ & 0.847715 & $8.87157 \times 10^{-7}$ \\
\hline 1. & 1. & $4.53456 \times 10^{-3}$ & 1. & $1.73788 \times 10^{-3}$ & 1. & $2.24424 \times 10^{-6}$ \\
\hline
\end{tabular}

TABLE 4: Solutions along with residual errors for RKF45, HPM, and LSHPM when $R=0.3, M_{g}=0.9, M_{p}=0.9$, and $\gamma=0.9$ in case of slip.

\begin{tabular}{lcccccc}
\hline$\xi$ & \multicolumn{2}{c}{ Fehlberg RK } & \multicolumn{2}{c}{ HPM } & \multicolumn{2}{c}{ LSHPM } \\
\hline 0. & Solution & Error & Solution & Error & Solution & Error \\
0.1 & 0. & $4.83512 \times 10^{-4}$ & 0. & 0. & 0. & 0.0731283 \\
0.2 & 0.0731283 & $5.03095 \times 10^{-5}$ & 0.0731173 & $9.14697 \times 10^{-4}$ & $0.14086 \times 10^{-7}$ \\
0.3 & 0.147841 & $7.32584 \times 10^{-6}$ & 0.14782 & $1.74106 \times 10^{-3}$ & 0.147841 & $2.519 \times 10^{-7}$ \\
0.4 & 0.225732 & $4.51413 \times 10^{-6}$ & 0.225703 & $2.39716 \times 10^{-3}$ & 0.225732 & $7.00476 \times 10^{-8}$ \\
0.5 & 0.308414 & $4.8655 \times 10^{-6}$ & 0.308379 & $2.81394 \times 10^{-3}$ & 0.308414 & $1.89255 \times 10^{-7}$ \\
0.6 & 0.397527 & $1.11029 \times 10^{-6}$ & 0.39749 & $2.94152 \times 10^{-3}$ & 0.397527 & $2.79031 \times 10^{-7}$ \\
0.7 & 0.494748 & $5.13492 \times 10^{-6}$ & 0.494712 & $2.75542 \times 10^{-3}$ & 0.494748 & $5.70387 \times 10^{-8}$ \\
0.8 & 0.601801 & $2.07476 \times 10^{-5}$ & 0.60177 & $2.26228 \times 10^{-3}$ & 0.601801 & $2.8116 \times 10^{-7}$ \\
0.9 & 0.720459 & $3.57011 \times 10^{-5}$ & 0.720436 & $1.50464 \times 10^{-3}$ & 0.720459 & $2.07976 \times 10^{-7}$ \\
1. & 0.852559 & $2.49738 \times 10^{-4}$ & 0.852546 & $5.64127 \times 10^{-4}$ & 0.852559 & $3.93393 \times 10^{-7}$ \\
\hline
\end{tabular}




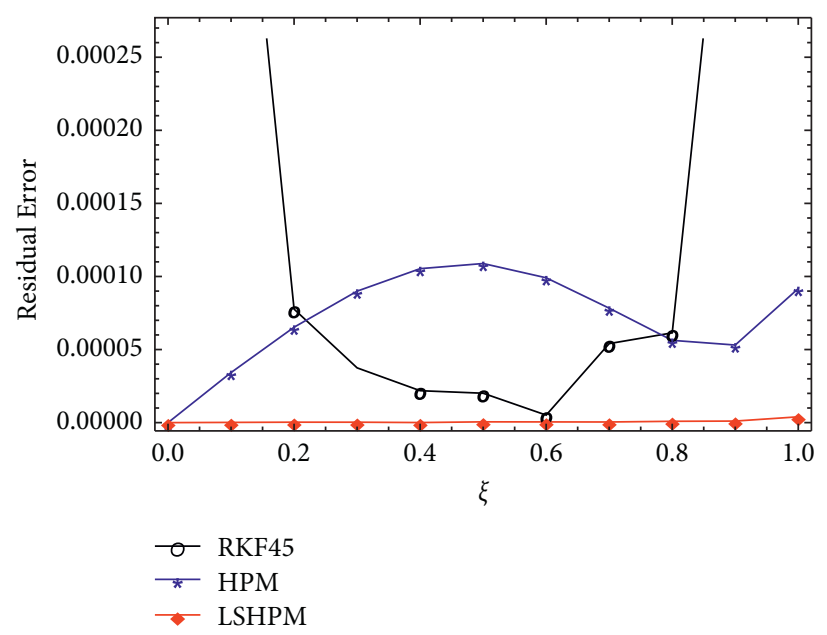

Figure 1: Comparison of errors for RKF45, HPM, and LSHPM when $R=0.5, M_{g}=1$, and $M_{p}=0.7$ in case of no slip.

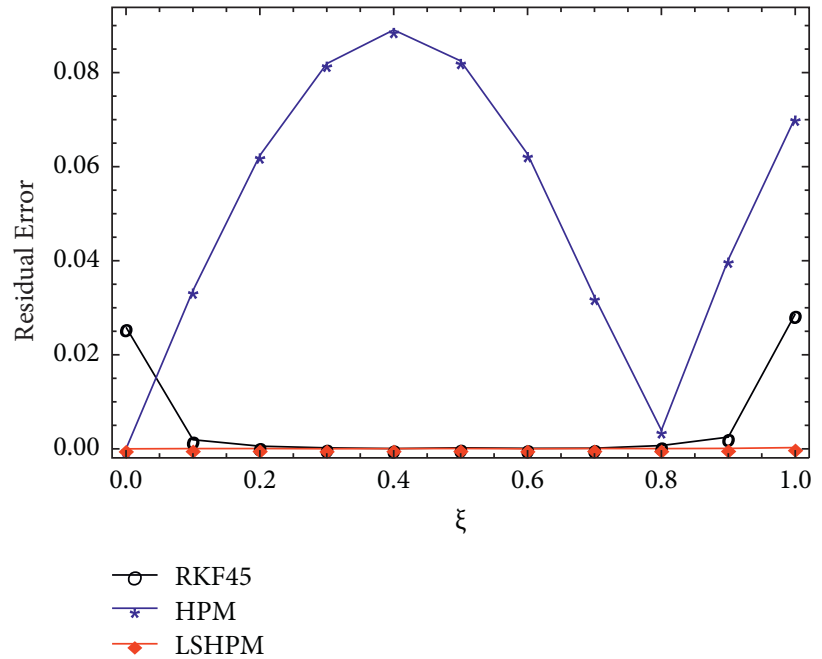

Figure 2: Comparison of errors for RKF45, HPM, and LSHPM when $R=1, M_{g}=1$, and $M_{p}=0.3$ in case of no slip.

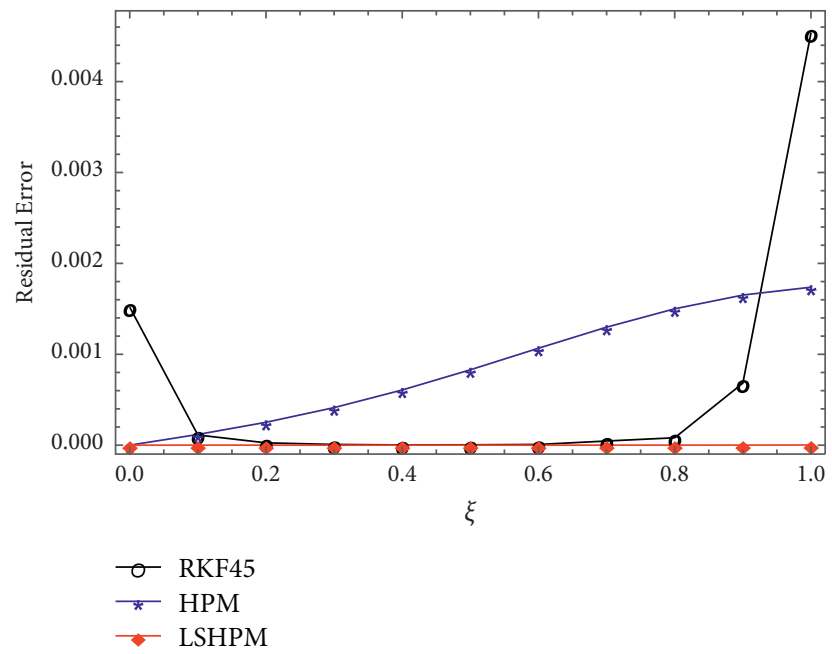

Figure 3: Comparison of errors for RKF45, HPM, and LSHPM when $R=0.5, M_{g}=1, M_{p}=0.8$, and $\gamma=0.9$ in case of slip. 


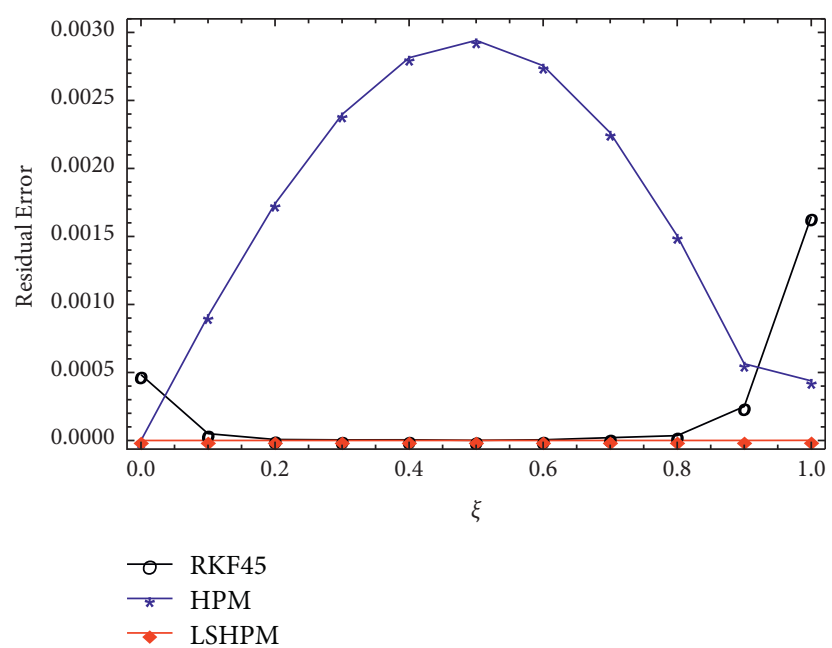

FIgURE 4: Comparison of errors for RKF45, HPM, and LSHPM when $R=0.3, M_{g}=0.9, M_{p}=0.9$, and $\gamma=0.9$ in case of slip.

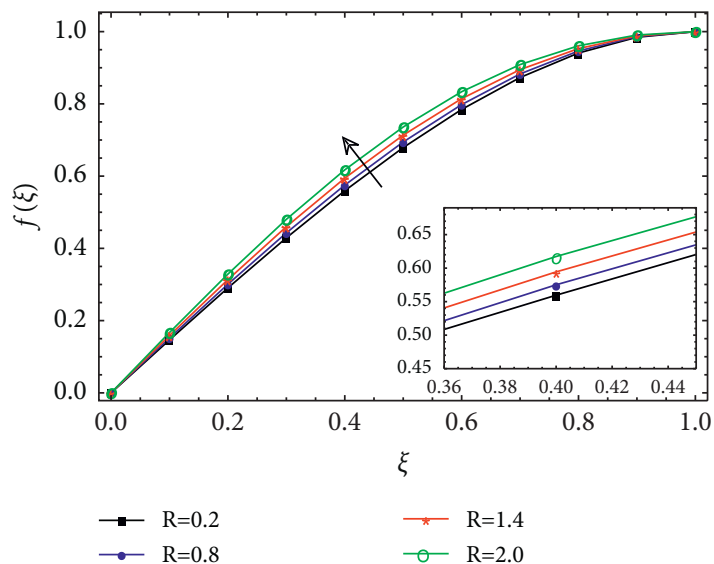

(a)

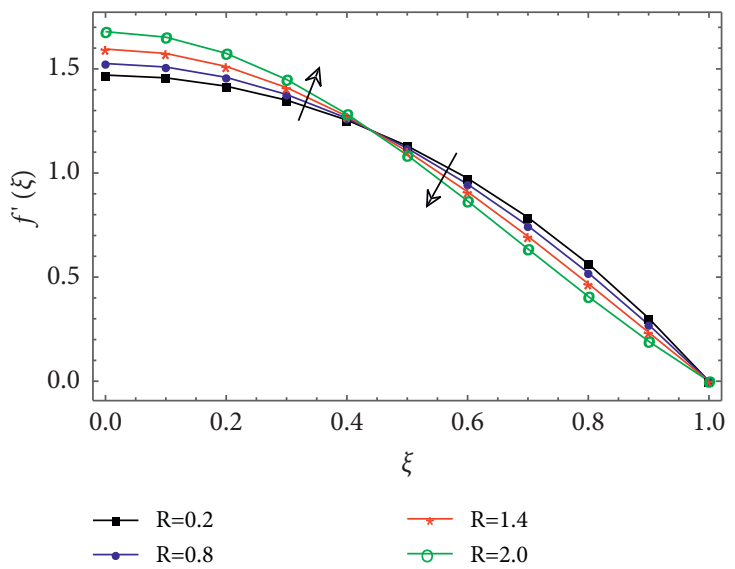

(b)

FIGURE 5: Effect of $R$ on the velocity profile keeping $M_{g}=1$ and $M_{p}=1$ fixed in case of no slip. (a) Normal component of velocity. (b) Axial component of velocity.

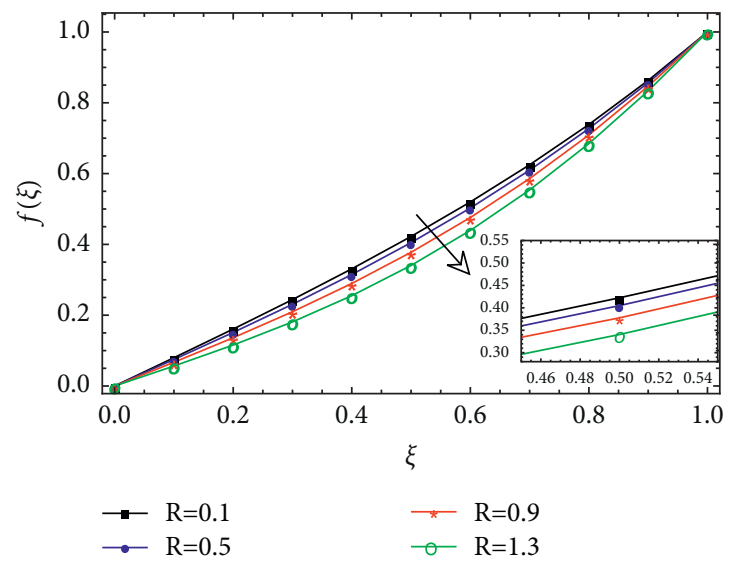

(a)

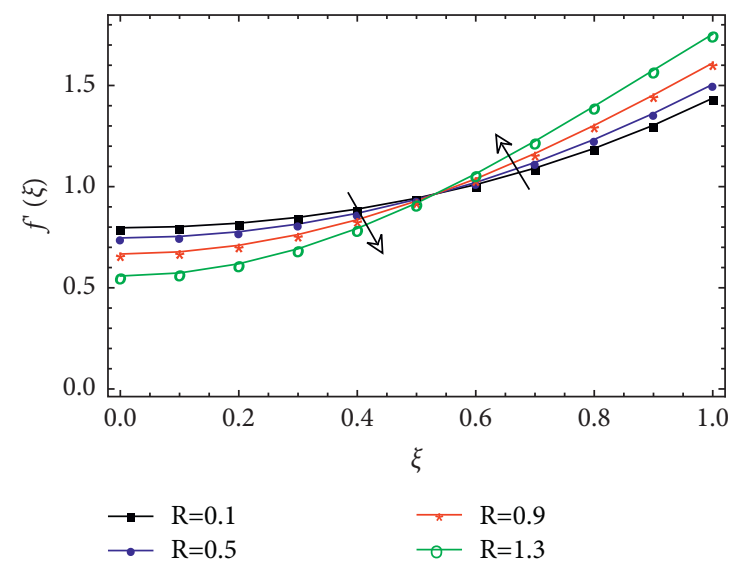

(b)

Figure 6: Effect of $R$ on the velocity profile keeping $M_{g}=1, M_{p}=1$, and $\gamma=1$ fixed in case of slip. (a) Normal velocity. (b) Axial velocity. 


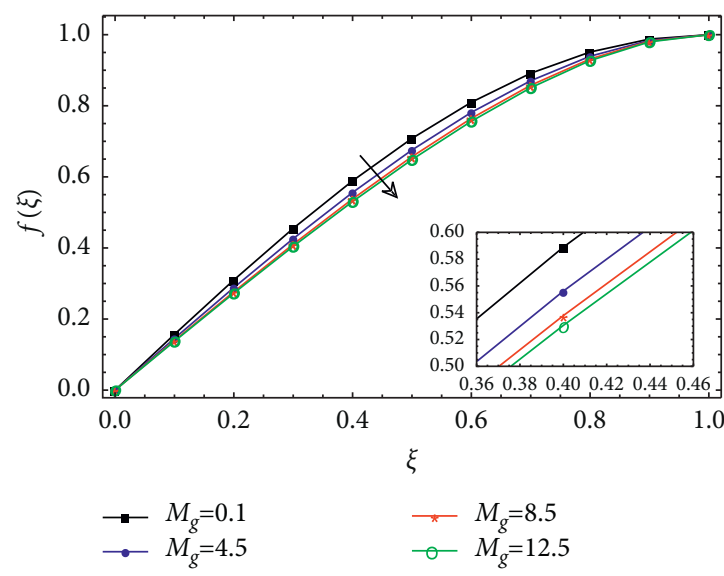

(a)

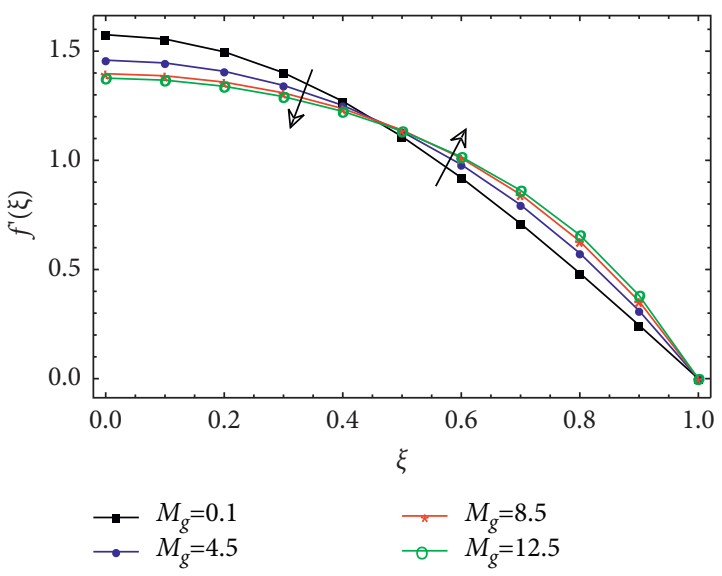

(b)

Figure 7: Effect of $M_{g}$ on the velocity profile keeping $R=1$ and $M_{p}=1$ fixed in case of no slip. (a) Normal component of velocity. (b) Axial component of velocity.

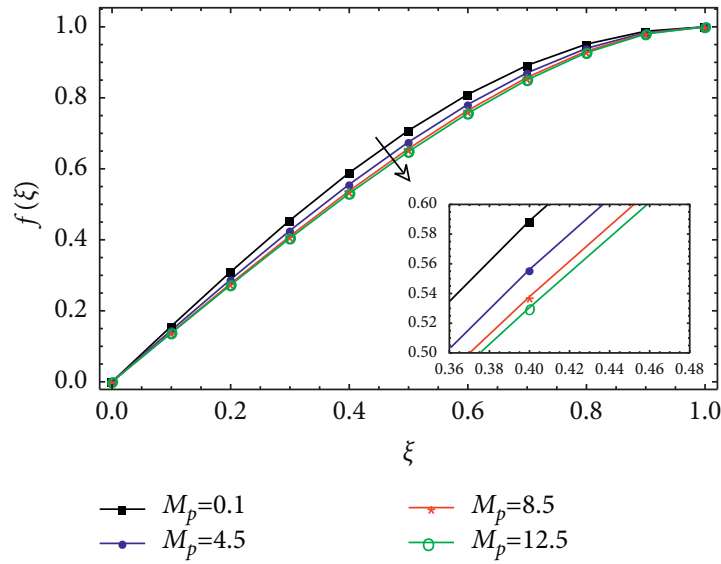

(a)

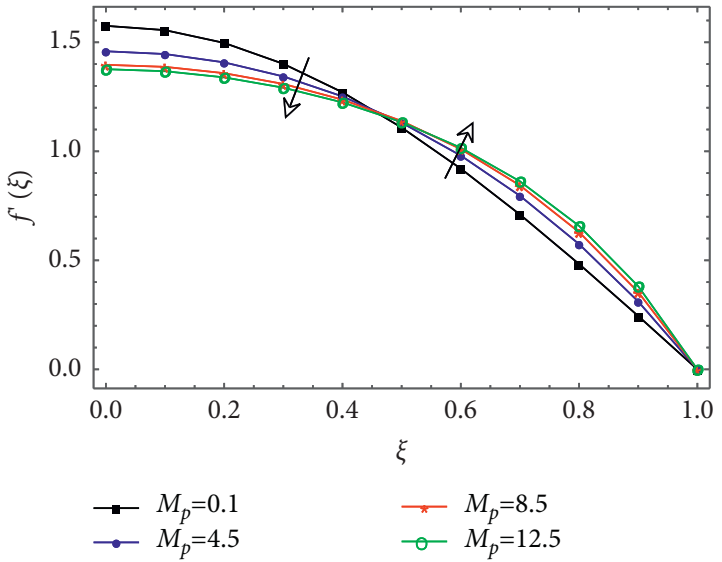

(b)

FIgURe 8: Effect of $M_{p}$ on the velocity profile keeping $R=1$ and $M_{g}=1$ fixed in case of no slip. (a) Normal component of velocity. (b) Axial component of velocity.

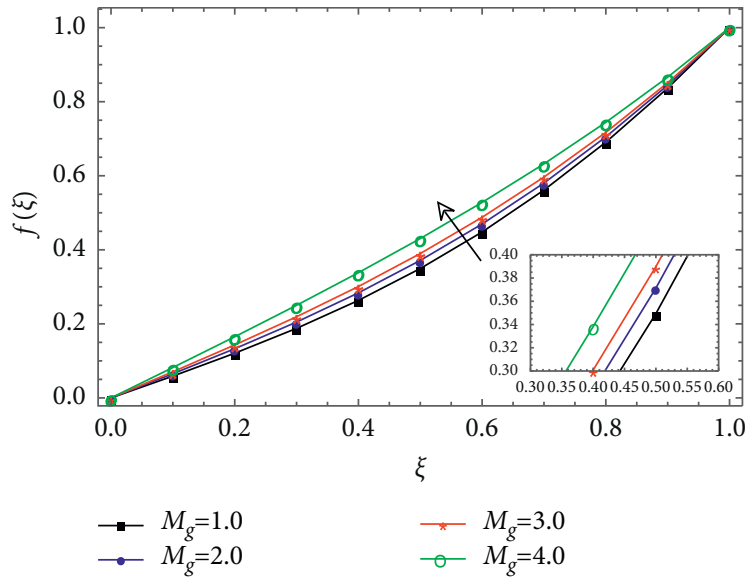

(a)

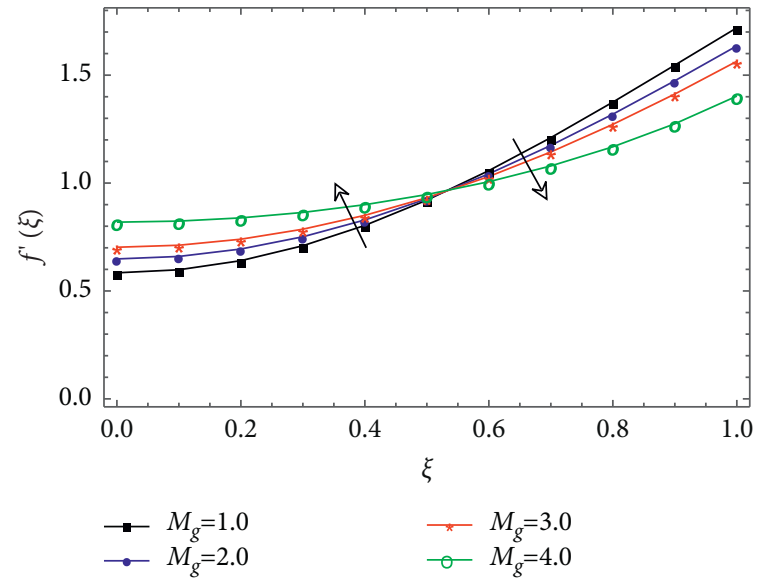

(b)

FIGURE 9: Effect of $M_{g}$ on the velocity profile keeping $R=1, M_{p}=0.1$, and $\gamma=1$ fixed in case of slip. (a) Normal component of velocity. (b) Axial component of velocity. 


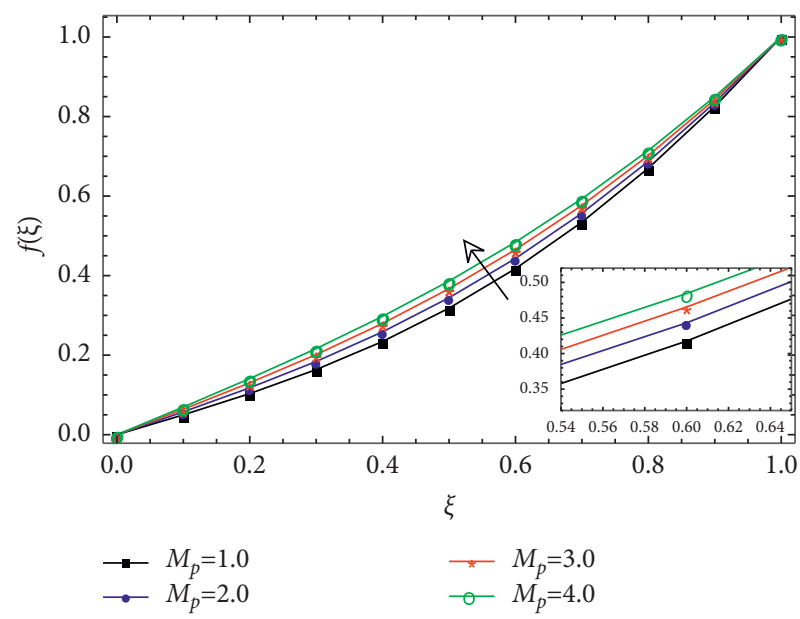

(a)

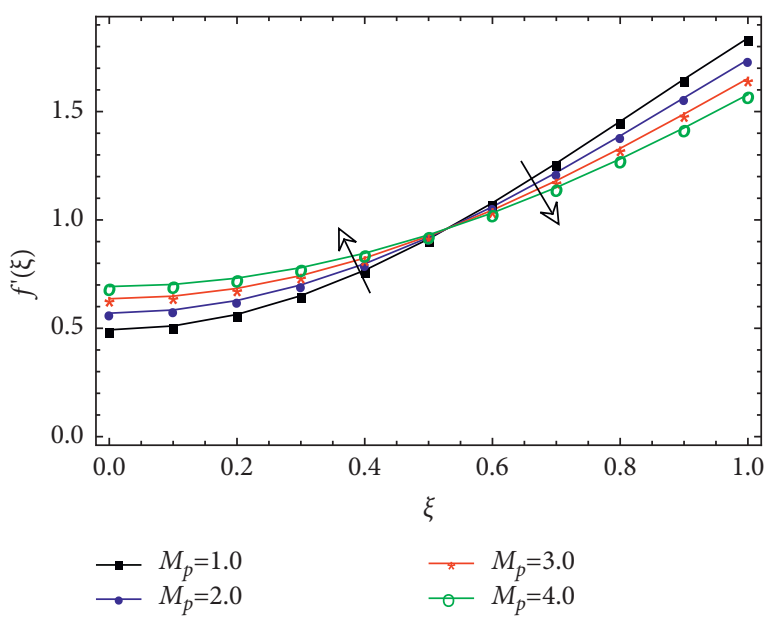

(b)

FIGURE 10: Effect of $M_{P}$ on the velocity profile keeping $R=1.5, M_{g}=1$, and $\gamma=1$ fixed in case of slip. (a) Normal component of velocity. (b) Axial component of velocity.

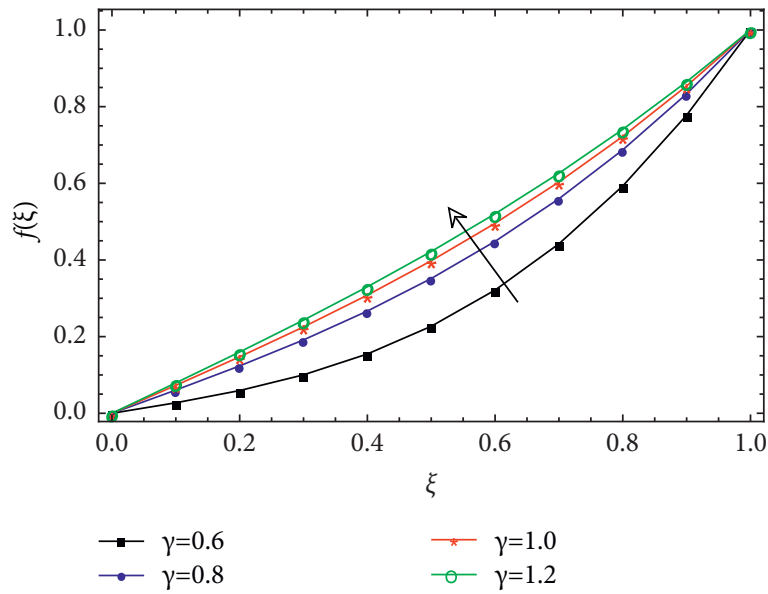

(a)

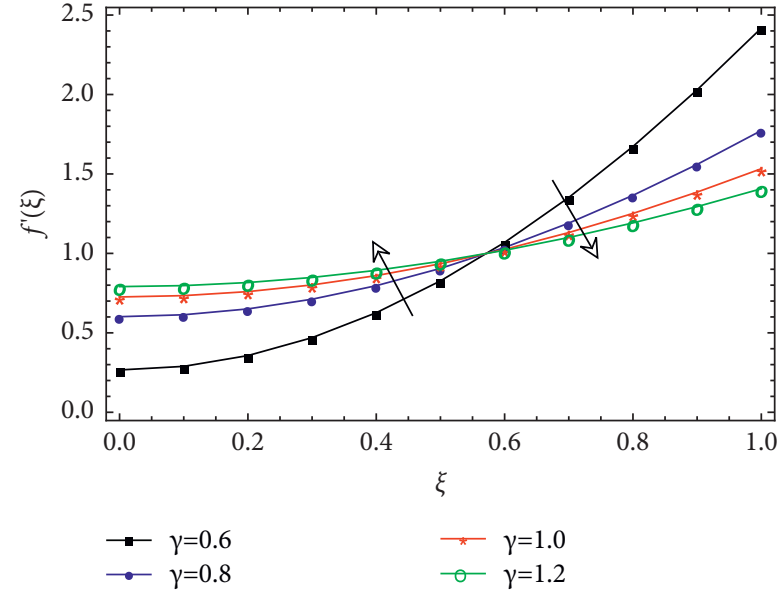

(b)

Figure 11: Effect of $\gamma$ on the velocity profile keeping $R=0.5, M_{g}=1$, and $M_{p}=0.5$ fixed in case of slip. (a) Normal component of velocity. (b) Axial component of velocity.

The convergence of LSHPM is also shown in Figures 1 and 2 in case of no slip and Figures 3 and 4 in case of slip at the boundary.

$$
\begin{aligned}
R\left(\xi, c_{3}, c_{4} c_{5}, c_{6}\right)= & 120 c_{3} \xi+840 c_{4} \xi^{3}+3024 c_{5} \xi^{5}+7920 c_{6} \xi^{7}-M_{p}\left(3\left(-1-4 c_{3}-6 c_{4}-8 c_{5}-10 c_{6}\right) \xi\right. \\
& \left.+20 c_{3} \xi^{3}+42 c_{4} \xi^{5}+72 c_{5} \xi^{7}+110 c_{6} \xi^{9}\right)-M_{g}\left(3\left(-1-4 c_{3}-6 c_{4}-8 c_{5}-10 c_{6}\right) \xi+20 c_{3} \xi^{3}+42 c_{4} \xi^{5}\right. \\
& \left.+72 c_{5} \xi^{7}+110 c_{6} \xi^{9}\right)-R\left(-\xi\left(3\left(-1-4 c_{3}-6 c_{4}-8 c_{5}-10 c_{6}\right)+60 c_{3} \xi^{2}+210 c_{4} \xi^{4}+504 c_{5} \xi^{6}+990 c_{6} \xi^{8}\right)\right. \\
& -3\left(3\left(-1-4 c_{3}-6 c_{4}-8 c_{5}-10 c_{6}\right) \xi\right.
\end{aligned}
$$




$$
\begin{aligned}
& \left.+20 c_{3} \xi^{3}+42 c_{4} \xi^{5}+72 c_{5} \xi^{7}+110 c_{6} \xi^{9}\right)-\left(3\left(-1-4 c_{3}-6 c_{4}-8 c_{5}-10 c_{6}\right) \xi+20 c_{3} \xi^{3}+42 c_{4} \xi^{5}+72 c_{5} \xi^{7}+110 c_{6} \xi^{9}\right) \\
& \left(1-c_{3}-c_{4}-c_{5}-c_{6}+\frac{1}{2}\left(1+4 c_{3}+6 c_{4}+8 c_{5}+10 c_{6}\right)+\frac{3}{2}\left(-1-4 c_{3}-6 c_{4}-8 c_{5}-10 c_{6}\right) \xi^{2}\right. \\
& \left.+5 c_{3} \xi^{4}+7 c_{4} \xi^{6}+9 c_{5} \xi^{8}+11 c_{6} \xi^{10}\right)+\left(3\left(-1-4 c_{3}-6 c_{4}-8 c_{5}-10 c_{6}\right)+60 c_{3} \xi^{2}+210 c_{4} \xi^{4}+504 c_{5} \xi^{6}+990 c_{6} \xi^{8}\right)\left(\left(1-c_{3}-c_{4}\right.\right. \\
& \left.\left.\left.-c_{5}-c_{6}+\frac{3}{2}\left(1+4 c_{3}+6 c_{4}+8 c_{5}+10 c_{6}\right)\right) \xi+\frac{3}{2}\left(-1-4 c_{3}-6 c_{4}-8 c_{5}-10 c_{6}\right) \xi^{3}+c_{3} \xi^{5}+c_{4} \xi^{7}+c_{5} \xi^{9}+c_{6} \xi^{11}\right)\right) .
\end{aligned}
$$

In addition, a graphical study has also been conducted to check the impact of different fluid parameters on the normal and axial velocity. Figures 5 and 6 show the effect of $R$ on the velocity profile in case of no slip and slip at the boundary. As the Reynolds number is the ratio of inertial forces to viscous forces within a fluid, it has the ability to calculate scaling effects and can be used to help predict fluid behavior on a larger scale. Due to this, normal velocity has shown an increase with an increase in $R$, while axial velocity decreases near the central axis and increases near the wall when no-slip boundary condition is employed. $R$ shows opposite effect when slip occurs at the boundary. Figures 7 and 8 present the effect of $M_{g}$ and $M_{p}$ on velocity profile in case of no slip. $M_{g}$ plays the role of a resistance contributed to by the magnetic pressure field component of Lorentz force. Due to this, the normal velocity decreases with an increase in $M_{g}$, whereas the axial velocity increases near the wall but decreases near the central axis of the channel. $M_{p}$ has an inverse relationship with the permeability constant $k$, and hence it shows similar behavior as $M_{g}$ when keeping other parameters fixed. $M_{g}$ and $M_{p}$ show opposite effect in slip case which can be seen in Figures 9-11.

\section{Conclusion}

In this article, an unsteady squeezing flow of MHD fluid passing through a porous medium has been modeled and analyzed considering the cases of slip and no slip at the boundaries using LSHPM. For validation purpose, the modeled nonlinear problems are also solved through HPM and Fehlberg-Runge-Kutta method. The obtained results show the effectiveness of LSHPM over other schemes. Obtained solutions clearly show that LSHPM is a more consistent scheme in terms of accuracy with less computational cost as compared to the other stated schemes and can be employed in various fields of science and engineering.

\section{Abbreviations}

$\tilde{u}$ : $\quad$ Velocity components along $x$-axis

$\tilde{v}$ : $\quad$ Velocity components along $y$-axis

$\sigma: \quad$ Electric conductivity

$\rho: \quad$ Density

$\nu: \quad$ Kinematic viscosity

$\widetilde{\omega}: \quad$ Vorticity function

$\mu_{0}: \quad$ Magnetic permeability

$\beta$ : $\quad$ Uniform magnetic field

$\begin{array}{ll}R: & \text { Reynolds number } \\ M_{g}: & \text { MHD parameter } \\ M_{p}: & \text { Porosity parameter } \\ h: & \text { Generalized pressure } \\ \xi: & \text { Dimensionless variable } \\ \gamma: & \text { Slip parameter } \\ \text { HPM: } & \text { Homotopy perturbation method }\end{array}$

LSHPM: Least squares homotopy perturbation method.

\section{Data Availability}

The data used to support the findings of this study are included within the article.

\section{Conflicts of Interest}

The authors declare that they have no conflicts of interest.

\section{References}

[1] Q. K. Ghori, M. Ahmed, and A. M. Siddiqui, "Application of homotopy perturbation method to squeezing flow of a Newtonian fluid," International Journal of Nonlinear Sciences and Numerical Stimulation, vol. 8, no. 2, pp. 179-184, 2007.

[2] X. J. Ran, Q. Y. Zhu, and Y. Li, “An explicit series solution of the squeezing flow between two infinite plates by means of the homotopy analysis method," Communications in Nonlinear Science and Numerical Simulation, vol. 14, no. 1, pp. 119-132, 2009.

[3] G. Domairry and A. Aziz, "Approximate analysis of MHD squeeze flow between two parallel disks with suction or injection by homotopy perturbation method," Mathematical Problems in Engineering, vol. 2009, Article ID 603916, 2009.

[4] J. A. Al-Saif and A. M. Jasim, "A novel algorithm for studying the effects of squeezing flow of a casson fluid between parallel plates on magnetic field," Journal of Applied Mathematics, vol. 2019, Article ID 3679373, 19 pages, 2019.

[5] M. J. Stefan, "Versuch Über die scheinbare adhäsion," Sitzungsberichte/Österreichische Akademie der Wissenschaften in Wien Mathematisch-Naturwissenschaftliche Klasse, vol. 69, pp. 713-721, 1874.

[6] E. R. Maki, D. C. Kuzma, and R. J. Donnelly, "Magnetohydrodynamic lubrication flow between parallel plates," Journal of Fluid Mechanics, vol. 26, no. 3, pp. 537-543, 1966.

[7] W. F. Hughes and R. A. Elco, "Magnetohydrodynamic lubrication flow between parallel rotating disks," Journal of Fluid Mechanics, vol. 13, no. 1, pp. 21-32, 1962.

[8] D. C. Kuzma, E. R. Maki, and R. J. Donnelly, "The magnetohydrodynamic squeeze film," Journal of Fluid Mechanics, vol. 19, no. 3, pp. 395-400, 1964. 
[9] N. A. M. Noor, S. Shafie, and M. A. Admon, "Heat and mass transfer on MHD squeezing flow of Jeffrey nanofluid in horizontal channel through permeable medium," PLoS One, vol. 16, no. 5, Article ID e0250402, 2021.

[10] N. S. Khashi'ie, I. Waini, N. M. Arifin, and I. Pop, "Unsteady squeezing flow of $\mathrm{Cu}-\mathrm{Al}_{2} \mathrm{O}_{3}$ /water hybrid nanofluid in a horizontal channel with magnetic field," Scientific Reports, vol. 11, no. 1, 2021.

[11] E. A. Hamza, "The magnetohydrodynamic squeeze film," Journal of Tribology, vol. 110, no. 2, pp. 375-377, 1988.

[12] E. A. Hamza, "The magnetohydrodynamic effects on a fluid film squeezed between two rotating surfaces," Journal of Physics D: Applied Physics, vol. 24, no. 4, pp. 547-554, 1991.

[13] S. Bhattacharyya and A. Pal, "Unsteady MHD squeezing flow between two parallel rotating discs," Mechanics Research Communications, vol. 24, no. 6, pp. 615-623, 1997.

[14] M. H. Hamdan, "An alternative approach to exact solutions of a special class of Navier-Stokes flows," Applied Mathematics and Computation, vol. 93, no. 1, pp. 83-90, 1998.

[15] M. H. Hamdan and F. M. Allan, "A note on the generalized beltrami flow through porous media," International Journal of Pure and Applied Mathematics, vol. 27, no. 4, p. 491, 2006.

[16] S. Islam, M. Raheel Mohyuddin, and C. Zhou, "Few exact solutions of non-Newtonian fluid in porous medium with hall effect," Journal of Porous Media, vol. 11, no. 7, 2008.

[17] M. Qayyum and H. Khan, "Behavioral study of unsteady squeezing flow through porous medium," Journal of Porous Media, vol. 19, no. 1, pp. 83-94, 2016.

[18] O. A. Bég, M. M. Rashidi, T. A. Bég, and M. Asadi, "Homotopy analysis of transient magneto-bio-fluid dynamics of micropolar squeeze film in a porous medium: a model for magneto-bio-rheological lubrication," Journal of Mechanics in Medicine and Biology, vol. 12, no. 3, 2012.

[19] K. S. Sorbie, "Depleted layer effects in polymer flow through porous media," Journal of Colloid and Interface Science, vol. 139, no. 2, pp. 299-314, 1990.

[20] S. Luk, R. Mutharasan, and D. Apelian, "Experimental observations of wall slip: tube and packed bed flow," Industrial \& Engineering Chemistry Research, vol. 26, no. 8, pp. 1609-1616, 1987.

[21] C. Neto, D. R. Evans, E. Bonaccurso, H.-J. Butt, and V. S. J. Craig, "Boundary slip in Newtonian liquids: a review of experimental studies," Reports on Progress in Physics, vol. 68, no. 12, pp. 2859-2897, 2005.

[22] M. Qayyum, H. Khan, and O. Khan, "Slip analysis at fluidsolid interface in MHD squeezing flow of casson fluid through porous medium," Results in Physics, vol. 7, pp. 732-750, 2017.

[23] R. F. Westover, "The significance of slip of polymer melt flow," Polymer Engineering \& Science, vol. 6, no. 1, pp. 83-89, 1966.

[24] M. Mooney, "Explicit formulas for slip and fluidity," Journal of Rheology, vol. 2, no. 2, pp. 210-222, 1931.

[25] R. Buscall, "Letter to the editor: wall slip in dispersion rheometry," Journal of Rheology, vol. 54, no. 6, pp. 1177-1183, 2010.

[26] J. H. He, "Homotopy perturbation technique," Computer Methods in Applied Mechanics and Engineering, vol. 178, no. 3-4, pp. 257-262, 1999.

[27] A. Kolyshkin and S. Nazarovs, "Stability of slowly diverging flows in shallow water," Mathematical Modelling and Analysis, vol. 12, no. 1, pp. 101-106, 2007.

[28] A. Krylovas and R. Čiegis, "Asymptotical analysis of one dimensional gas dynamics equations," Mathematical Modelling and Analysis, vol. 6, no. 1, pp. 117-128, 2001.
[29] A. H. Nayfeh, Introduction to Perturbation Techniques, John Wiley \& Sons, New York, NY, USA, 1981.

[30] A. M. Siddiqui, S. Irum, and A. R. Ansari, "Unsteady squeezing flow of a viscous MHD fluid between parallel plates, a solution using the homotopy perturbation method," Mathematical Modelling and Analysis, vol. 13, no. 4, pp. 565-576, dec 2008.

[31] P. Singh, V. Radhakrishnan, and K. A. Narayan, "Squeezing flow between parallel plates," Ingenieur-Archiv, vol. 60, no. 4, pp. 274-281, 1990. 